EPJ Web of Conferences 67, 02070 (2014)

DOI: 10.1051 /epjconf / 20146702070

(C) Owned by the authors, published by EDP Sciences, 2014

\title{
Flow in data racks
}

\author{
Lukáš Manoch $^{1, \mathrm{a}}$, Jan Matěcha $^{1, \mathrm{~b}}$, Jan Novotný ${ }^{1, \mathrm{c}}$, Jiří Nožička ${ }^{1, \mathrm{~d}}$, and Petr Pohan ${ }^{2, \mathrm{e}}$ \\ 1 CTU in Prage, Faculty of Mechanical Engineering, Department of Fluid Dynamics and Thermodynamics, Technická 4, \\ Praha 6, Czech Republic \\ 2 Conteg, spol. s r.o., Na Vítězné pláni 1719/4, Praha 4, Czech Republic
}

\begin{abstract}
This paper deals with the flow in data racks. The aim of this work is to find a new arrangement of elements regulating the flow in the data rack so that the aerodynamic losses and the recirculation zones were minimized. The main reason for solving this problem is to reduce the costs of data racks cooling. Another problem to be solved is a reverse flow in the servers, thus not cooled, occuring due to the underpressure in the recirculation zones. In order to solve the problem, the experimental and numerical model of $27 \mathrm{U}$ data rack fitted with 10 pieces of server models with a total input of $10 \mathrm{~kW}$ was created. Different configurations of layout of elements affecting the flow in the inlet area of the data rack were compared. Depending on the results achieved, design solutions for the improvement of existing solutions were adopted and verified by numerical simulations.
\end{abstract}

\section{Aim/Motivation}

The aim of the work was to reduce overall cooling costs in data centers. There are many studies and ways of dealing with the problem at the level of the whole data centers, or the elements constituting data centers. This paper focuses on the level of data racks (27U) and servers (1U), specifically on the suction area of servers in the data rack. This include modification of the current state or equipment in the direction to reduce the effects of undesirable increase of energy consumption necessary for effective cooling of fitted components - the servers, in this particular case. The above mentioned undesirable effects can be described e.g. as recirculation zones, under-pressure areas or areas of flow separation.

\section{Current state}

Nowadays, there is an enormous increase in the number of data centers. In relation to this phenomenon, the techniques focused on reducing the energy consumption required for the operation of the data center are being developed [1] [2] [3]. The introduction of a completely new technique is usually very expensive and often impossible for already operating data centers. Due to the impossibility of implementation of a new technique or technology for the existing data centers, it is necessary to adjust or modify the current equipment. This approach has great limitations and the changes made must be easy to implement, if possible, without restriction of data rack operation.

There is a whole range of methods of cooling the data racks. From simple cooling of the whole data center room

\footnotetext{
a e-mail: lukas.manoch@fs.cvut.cz

b e-mail: jan.matecha@fs.cvut.cz

c e-mail: jan.novotny@fs.cvut.cz

d e-mail: jiri.nozicka@fs.cvut.cz

e e-mail: p.pohan@conteg.cz
}

(a very ineffective and energy consuming method) to separated hot and cold aisles, where the suction and exhaust areas of servers are completely separated, thus leading to no mixing of hot and cold air. Another method of cooling is the creation, with the help of a side cooler and data rack, of closed area with a very effective cooling but higher cost of purchase.

One of the methods used most often is when the cold air is supplied to the lower part of the data rack and then enters the suction part to servers. Hot air is then collected from the room and cooled again. This type of cooling is a certain variation of the hot and cold aisle since there is no contact of hot and cold air. This kind of cooling was chosen to be the main subject of the paper as it is the most easily modifiable.

\section{Data rack modification}

In case of the type of cooling considered, the modification of data rack is relatively easy, as there is no direct intervention into the construction itself, but it is a modification or addition of other elements in the unused part of the data rack.

In the lower part of the data rack, at the cold air inlet, the cold air is directed to the suction area of servers with the help of a current corrector (figure 3 ). For the existing data rack, this element created both barrier to avoid cold air leakage to other data rack parts, and should somehow correct the direction of the inlet cold air flow. As a result of an improperly chosen geometry of the element, air flow separation occurred in the lower part of the data rack to the height of several $1 \mathrm{U}$ servers. In the area of that separation, the pressure dropped to such a value, that within the first few $1 \mathrm{U}$ servers air flow reverse occurred and hot air was sucked to the "cold" aisle area from the rear part of the data rack. Appropriate modification of the geometry shape can almost eliminate this problem, or reduce at least the flow separation and eliminate the formation of the underpressure area. 
Modifications have been made for two sizes of the cold air inlet to the suction area. The sizes were $1 \mathrm{U}$ and $3 \mathrm{U}$ where 1U (1 Unit) is a unit of measure based on American National Standard and its dimension is the multiple of 1.75 inches, i.e. $1.75 \cdot 25.4=44.45 \mathrm{~mm}$.

The modification was based on the current state and only adjusted the geometry of already used element. The implementation for already operating data centers is very simple and can be carried out under full operation.

The other modification leading to improvement of the air inlet to the suction area in the data rack is the use of data rack sides, which may serve as a side cooling zone. For such usage it is necessary to add additional elements to the lower part of the data rack for correct cold air supply. This solution will ensure the usage of the whole bottom part, or the lower part of the data rack. It is also necessary to add a wall preventing the cold air from entering the servers area. This will provide creation of space going over the whole length of the data rack, through which the cold air can be conducted to the suction area of the data rack. In view of the flow nature it is impossible to consider the whole space but only its proportional part with a recirculation zone assumed in the farther upper corner. But the zone is already included in the design, thus having no major impact on the final solution. At this point, another possible modification is outlined - limitation of entry of cold air to the recirculation zone.

The second modification, the "side cooling zone" solution (figure 4), should lead to better cooling of servers in the upper part of the data rack. If we consider the case when data rack is fully fitted with $1 \mathrm{U}$ servers, an imperfect cooling of servers in the upper part of the data rack can occur due to relatively small space in the suction area of data rack and full fitting. In the paper, we consider a $27 \mathrm{U}$ data rack that is fitted with ten $1 \mathrm{U}$ servers models. It is not the most critical model situation that would occur if the $42 \mathrm{U}$ data rack fully fitted with $1 \mathrm{U}$ servers was used.

Both designed modifications were modeled with the help of 3D CAD software in the first phase and placed into the real CAD model of the $27 \mathrm{U}$ data rack geometry. The reason for the model inclusion was the fact that all the measurement was carried out on real $27 \mathrm{U}$ data rack.

A numerical simulation had been performed before the particular modified elements were produced in order to find optimal shape for the corrector geometry both for $1 \mathrm{U}$ and $3 \mathrm{U}$. The modification was based on observing the size and point of the flow separation and its subsequent minimization.

\section{Numerical simulation}

For numerical simulation, a simplified geometric model of the suction area of the data rack up to the area of the cold air inlet was created. The geometric model was simplified at the level of bolted joints, holes for bolted joints, grounding and of closing mechanism. For all these elements, the influence of the flow field was not assumed at a scale greater than negligible. As mentioned above, the model represented a $27 \mathrm{U}$ data rack and was evenly fitted with ten server models, thus using the whole height of the data rack. In order to obtain the reference values, the models of current design were created, for which the numerical simulation was carried out and the same parameters as for the modified versions were evaluated.

\subsection{Computational mesh}

The mesh for individual models was created from six-wall elements, in the whole volume of the mesh, including the area of boundary layer. The mesh was formed by the same procedure for each model and the same amount of elements was used for particular modifications of current designs in order to eliminate the errors as a result of other mesh. All models solved had a common symmetry plane. This plane was used only for mesh generation due to elimination of error caused by asymmetrically formed mesh. The mesh was created in the Beta ANSA v13 program and subsequently exported in the format for ANSYS Fluent solver.

\subsection{Solver setup}

Numerical simulation of 3D problem was performed in the commercial solver ANSYS Fluent v14. Uniform boundary conditions were defined for solution of all the models above. It applied to pressure boundary condition for suction points of servers, i.e. for each of ten points a negative pressure value simulating suction effect of fans was defined. The value of the boundary condition was set to $-100 \mathrm{~Pa}$ (this value is related to atmospheric pressure the value of which was $101325 \mathrm{~Pa}$ ). This boundary condition can be considered as an outlet boundary condition. For the inlet boundary condition, which was represented by geometry at the bottom of the data rack, atmospheric pressure was defined. For the remaining geometric elements a boundary condition with zero velocity was defined.

For the solution itself the isothermal, viscous and incompressible flow was considered. A pressure-based algorithm was used. For the spatial discretization an upwind scheme of second order was used and only steady solution was considered.

The given flow was assumed to have turbulent character, therefore a two-equation turbulence $k$ - epsilon model was chosen - modification Realizable. The flow near the walls was solved using the wall functions according to corresponding parameter $y^{+}$.

Several check points, monitors, were set, in which the stabilization of the monitored variables was being observed.

After the numerical verification that the modifications to the geometry of the current corrector are consistent with the assumption, the modified correctors were made and incorporated to the experimental setup.

\section{Experimental setup}

The experimental setup was made up of three independent parts (figure 1). $27 \mathrm{U}$ data rack in ten modified and two existing versions was being measured. The data rack modifications were performed at the level of individual elements rather than at the level of the whole data rack. Data rack was fitted with 10 server models with power input of 1 $\mathrm{kW}$. For each of the server models the power of the fan 

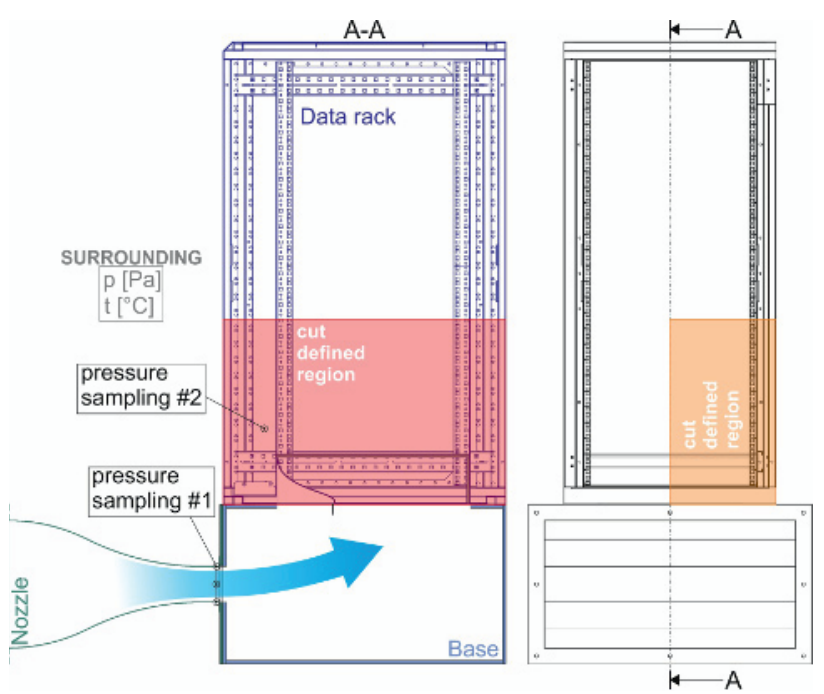

Fig. 1. Scheme used for experiment measurement.

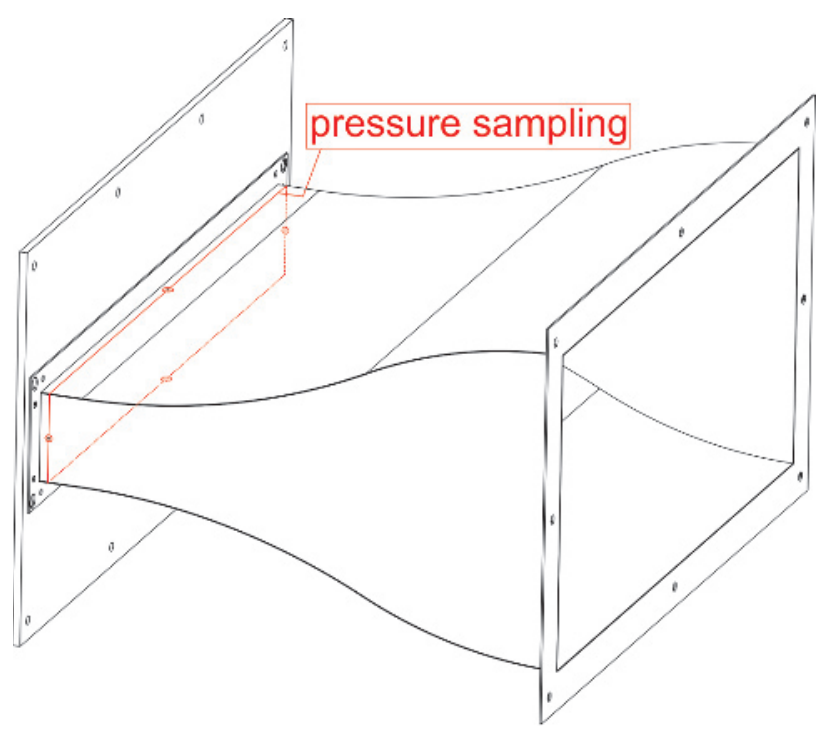

Fig. 2. Geometry of nozzle with positions for pressure sampling.

field, which consisted of eight fans, could be adjusted independently. Thermal load of the server models was not considered within the whole experimental measurement. Another element of the experimental setup - a nozzle (figure 2) - was designed for the measurement, which was equipped with four pressure taps along the perimeter at the outlet. The data rack was placed on a box- the last element of the experimental setup - to which the nozzle was attached. This box represented space that is situated in real data center, in which the cold air is being conducted. For all 12 compilations of the experimental setup 5 different powers on fan fields for individual server models were measured subsequently. These were $20 \%, 40 \%, 60 \%, 80 \%$ and $100 \%$ of power of the fan field. All the server models were simultaneously set to the same power. The mass flow throughout the whole setup was the main parameter of the whole setup to be evaluated. The parameter necessary for mass flow calculation was recorded with the help of pressure transducers during the entire measurement and subsequently its mean value was used for the calculation.

For each of the measured variants a zero pressure reading $[P a]$ was performed at the beginning and end of the
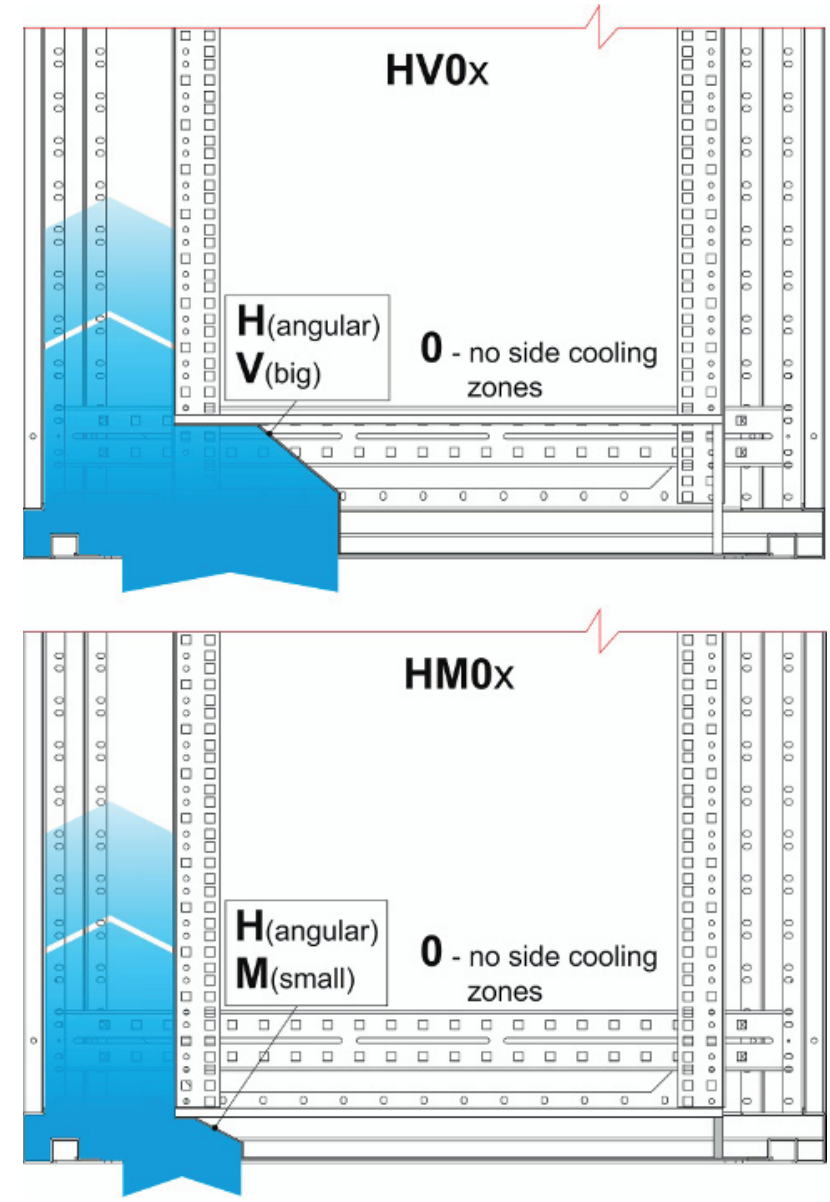

Fig. 3. Original used geometry and position for flow corectors.

measurement itself. The readings were performed due to measurement of values in the range of tens of $P a$ and the shift of zero reading at the end of the measurement in order of units of $P a$ would constitute an error in order of tens of $\%$. If the zero pressure readings differed by more than $0.5 \mathrm{~Pa}$ absolutely at the beginning and at the end of the measurement, the measurement was repeated.

The individual measured variants were labeled with a four-sign code (KM0A for example):

\section{First position}

- K - rounded deflector

- H - angular deflector

2. Second position

- M - small deflector

- V - big deflector

3. Third position

- 0 - without side cooling zones

- M - small side cooling zones

- V - big side cooling zones

4. Fourth position

- A - brace at the original place

- B - brace shifted upwards

- C - taped-over holes 


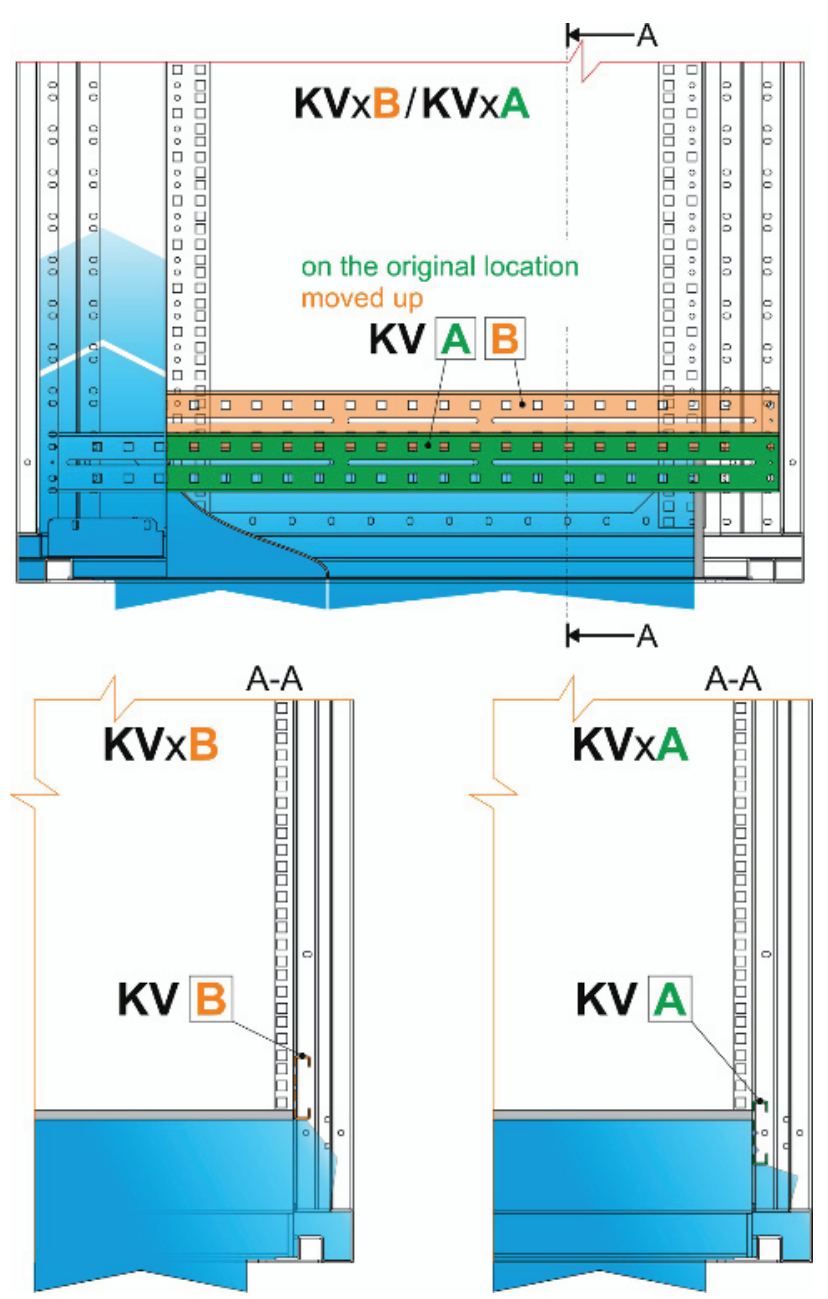

Fig. 4. Side cooling zones configuration for different position of side bar.

\section{Experimental setup}

In order to calculate the required mass flows $\dot{m}\left[\mathrm{~kg} \mathrm{~s}^{-1}\right]$ the following equation was used:

$$
\dot{m}=\rho A v,
$$

where the Bernoulli equation was used for defining the necessary additional variables:

$$
\frac{p_{0}}{\rho}+g h+\frac{v_{0}^{2}}{2}=\frac{p_{1}}{\rho}+g h+\frac{v_{1}^{2}}{2}+\frac{v_{1}^{2}}{2} \xi_{\text {nozzle }},
$$

where the indexes $i=0,1$ of pressure $p_{i}[P a]$ and velocities $v_{i}\left[m s^{-1}\right]$ represent the inlet, or outlet from the nozzle and the member $\xi_{\text {nozzle }}$ [1] represents the minor loss of the nozzle. Furthermore, during the measurement the temperature $t\left[{ }^{\circ} \mathrm{C}\right]$ at the nozzle inlet was being recorded, from which the density $\rho\left[\mathrm{kg} \mathrm{m}^{-3}\right]$ according to the equation of state of ideal gas was additionally calculated

$$
\rho=\frac{p}{r T},
$$

with specific gas constant $r\left[\mathrm{~J} \mathrm{~kg}^{-1} \mathrm{~K}^{-1}\right]$ and temperature $T[K]$. The relation between the velocities was expressed by the continuity equation, defined as:

$$
A_{0} v_{0}=A_{1} v_{1},
$$

where particular planes $A_{i}\left[\mathrm{~m}^{2}\right]$ are related to the corresponding places.
Table 1. Measured configurations with $100 \%$ of fan performance

\begin{tabular}{llllll}
\hline Label & $p b$ & $d p_{\text {nozzle }}$ & $p$ & $T$ & $\dot{m}$ \\
\hline & {$[P a]$} & {$[P a]$} & {$[P a]$} & {$\left[{ }^{\circ} \mathrm{C}\right]$} & {$\left[\mathrm{kg} \mathrm{m}^{-3}\right]$} \\
\hline KM0A & 98000 & 17.7 & -116.5 & 18.0 & 0.45 \\
KV0A & 97200 & 21.0 & -87.4 & 19.4 & 0.49 \\
HM0A & 97200 & 16.6 & -119.5 & 19.4 & 0.43 \\
HV0A & 97200 & 18.1 & -97.1 & 19.8 & 0.45 \\
KVVA & 97400 & 25.0 & -60.6 & 20.8 & 0.53 \\
KMVA & 97100 & 22.8 & -86.4 & 19.5 & 0.51 \\
KVVB & 99760 & 27.0 & -55.0 & 22.9 & 0.56 \\
KMVB & 99450 & 22.8 & -93.1 & 21.6 & 0.51 \\
KMMB & 99050 & 22.2 & -83.4 & 22.3 & 0.50 \\
KVMB & 99420 & 26.8 & -57.8 & 21.9 & 0.56 \\
KVMC & 99760 & 27.9 & -60.5 & 22.5 & 0.57 \\
KMMC & 99430 & 21.3 & -88.6 & 21.0 & 0.50 \\
KMVB & 99450 & 22.8 & -93.1 & 21.6 & 0.51 \\
KVMB & 99420 & 26.8 & -57.8 & 21.9 & 0.56
\end{tabular}

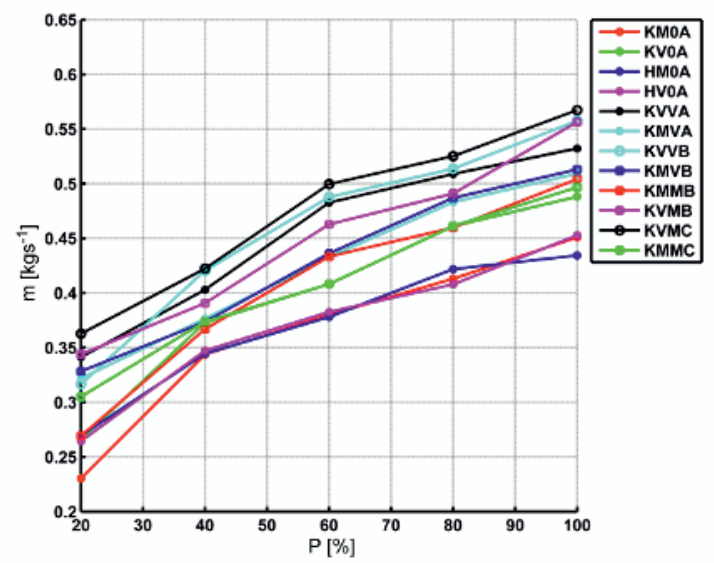

Fig. 5. Mass flow rate for each configuration and different fans performance $\mathrm{P}$.

\section{Results}

For the variants mentioned above, a power spectrum of fans were measured and the mass flows and pressure evaluated (figure 5, figure 6). All results shown that modifications made on flow correctors and addition side cooling zones increased mass flow rate throuht system. The biggest difference is about $20 \%$ according to reference states, and as a reference states are considered current states, as we can see in table 1 . For each measured case similar curve trend can be seen (figure 5). Modifications shifts curve just up without significant curve change. We can explain this by that we modified geometry and not whole model. For correctors modfications most of cross sections in the flow path stayed preserved. From this point of view we did not expect positive huge mass flow rate jump. Side cooling zones were added another volumes as a secondary flow path but the main reason were to supply more air to lower placed servers to eliminate back flow effect.

There were also measured pressure difference with and without using nozzle as we can see in figure 7. As can be seen the pressure lost for nozzle was form $10 \mathrm{~Pa}$ to $20 \mathrm{~Pa}$ for corresponding fan performance. 


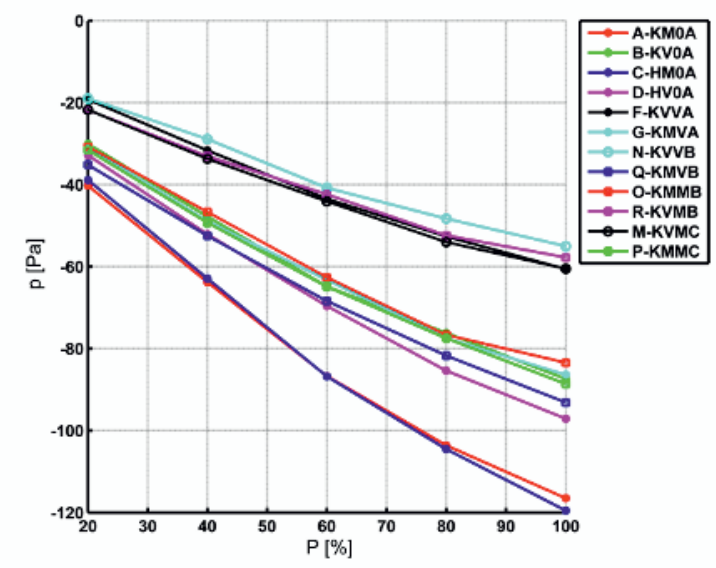

Fig. 6. Pressure variation for each configuration and different fans performance $P$.

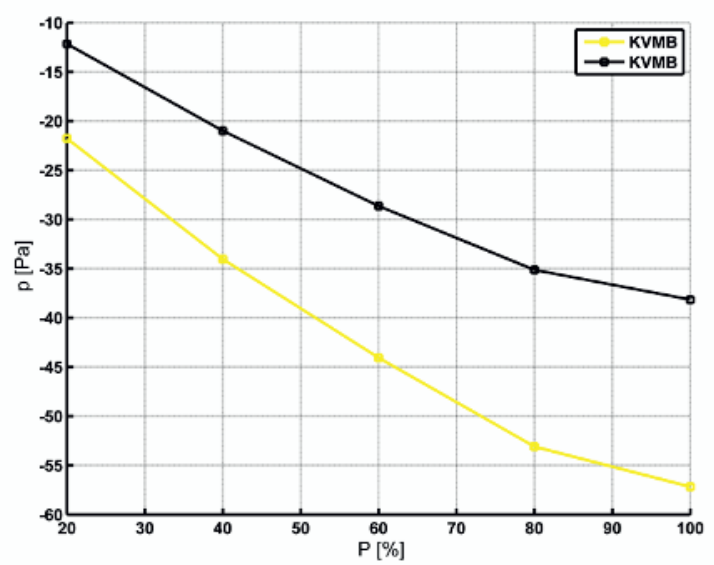

Fig. 7. Pressure variation for measurement with and without nozzle with different fans performance P. (black - with nozzle, yellow - without nozzle)

\section{Conclusion}

For the given compilation and modified geometry of correctors, it was proven that there is an increase in the mass flow throughout the whole setup. The impact of the side cooling zones was measured and evaluated leading to better distribution of air to the server suction area.

In case of modifying only the correctors an increase of mass flow throughout the whole setup was verified and should lead to reduction of costs connected with air distribution to the data rack. This modification is implementable in the current designs, even under full operation of the data rack. The addition of side cooling zones can further improve the air distribution to the servers but this kind of implementation is not easy to perform and it is more of an evolutionary element of the current designs.

\section{Acknowledgment}

Project (TA01010184 / Research and development solutions data racks, cooling and transport systems for data centers) is / was solved with the financial support of TA ČR.

\section{References}

1. L. Manoch, J. Nožička and P. Pohan, EPJ Web of Conferences, Volume 25, (2012)

2. L. Manoch, J. Novotný and L. Nováková, $22^{\text {nd }}$ International Symposium on Transport Phenomena, (2011)

3. L. Manoch, J. Matěcha and P. Pohan, EPJ Web of Conferences, Volume 45, (2013) 